\title{
Evaluation of models in the 2016 Cryo-EM Model Challenge
}

Andriy Kryshtafovych ${ }^{1}$, Catherine L. Lawson ${ }^{2}$, Wah Chiu ${ }^{3}$

${ }^{1}$ Genome Center, University of California, Davis, 451 Health Sciences Drive, Davis, CA 95616, USA

2 Center for Integrative Proteomics Research and Research Collaboratory for Structural Bioinformatics, Rutgers, The State University of New Jersey, 174 Frelinghuysen Road, Piscataway, NJ 08854, USA

3 Department of Bioengineering, Microbiology and Immunology and Photon Science, Stanford University, James H. Clark Center, MC5447, 318 Campus Drive, Stanford, CA 94305-5447

An evaluation system and a web infrastructure were developed for the second (2016) cryo-EM model challenge. The evaluation system includes tools to validate stereo-chemical plausibility of submitted models, check their fit to the corresponding density maps, estimate their overall and per-residue accuracy, and assess their similarity to reference cryo-EM or X-ray structures as well as other models submitted in this challenge. The web infrastructure provides a convenient interface for analyzing models at different levels of detail. It includes interactively sortable tables of evaluation scores for different subsets of models and different sublevels of structure organization, and a suite of visualization tools facilitating comparative model analysis. The results are publicly accessible at http://model-compare.emdatabank.org. 